\title{
Avaliação dos ligamentos acromioclavicular, coracoclaviculares e coracoclavicular medial na luxação acromioclavicular
}

\section{Acromioclavicular, Coracoclavicular and Medial Coracoclavicular Ligaments Assessment in Acromioclavicular Dislocation}

\author{
Rômulo Brasil Filho ${ }^{1}$ Marcelo Mamede de Freitas ${ }^{10}$ Rafael Henrique Rangel Nunes ${ }^{10}$ \\ Antonio Carlos Tenor Junior ${ }^{1}$ Miguel Pereira da Costa ${ }^{1}$ Rodrigo de Araújo Roberto ${ }^{1}$
${ }^{1}$ Hospital do Servidor Público Estadual de São Paulo (HSPE), IAMSPE, São Paulo, SP, Brasil
Rev Bras Ortop 2021;56(6):777-783.
Endereço para correspondência Marcelo Mamede de Freitas, MD, Hospital do Servidor Público Estadual de São Paulo, Rua Pedro de Toledo, 1800, São Paulo, São Paulo, 04039-901, Brasil (e-mail: marcelomamede@hotmail.com).

\section{Resumo \\ Palavras-chave \\ - articulação acromioclavicular \\ - luxações articulares \\ - anatomia \\ - dissecação}

Objetivo Estudar a anatomia do ligamento coracoclavicular medial e avaliar a contribuição do ligamento acromioclavicular, coracoclaviculares e coracoclavicular medial na estabilidade da articulação acromioclavicular.

Métodos Foram dissecados 26 ombros de 16 cadáveres frescos, posicionados em decúbito dorso-horizontal, com um coxim de $15 \mathrm{~cm}$ de altura entre as escápulas. Realizou-se uma via deltopeitoral estendida proximal e medialmente. Realizou-se dissecção por planos e identificação dos ligamentos. Realizou a medida da distância acromio-clavicular e coracoclavicular usando pontos previamente demarcados com paquímetro milimetrado. Em seis dessas amostras foi realizado estudo biomecânico. Seccionando, nesta ordem, o ligamento acromioclavicular, os coracoclaviculares e o ligamento coracoclavicular medial com uma força cefálica de $20 \mathrm{~N}$ foi aplicada na clavícula lateral. Foi medida a distância acromio-clavicular e coracoclavicular em cada uma das etapas de secção dos ligamentos.

Resultados A média de comprimento do ligamento coracoclavicular medial foi de $48,9 \mathrm{~mm}$ e a média de largura, de $18,3 \mathrm{~mm}$ no lado direito. No esquerdo, a média de comprimento foi de $48,65 \mathrm{~mm}$ e a média da largura, $17,3 \mathrm{~mm}$. Após a secção dos ligamentos acromioclaviculares, coracoclaviculares, com a secção do ligamento

Trabalho desenvolvido no Hospital do Servidor Público Estadual de São Paulo - HSPE - IAMSPE, São Paulo, SP, Brasil.

recebido

29 de Janeiro de 2020

aceito

16 de Setembro de 2020
DOI https://doi.org/

10.1055/s-0040-1719088. ISSN 0102-3616. (c) 2021. Sociedade Brasileira de Ortopedia e Traumatologia. All rights reserved.

This is an open access article published by Thieme under the terms of the Creative Commons Attribution-NonDerivative-NonCommercial-License, permitting copying and reproduction so long as the original work is given appropriate credit. Contents may not be used for commercial purposes, or adapted, remixed, transformed or built upon. (https://creativecommons.org/ licenses/by-nc-nd/4.0/)

Thieme Revinter Publicações Ltda., Rua do Matoso 170, Rio de Janeiro, RJ, CEP 20270-135, Brazil 


\begin{abstract}
Keywords

- acromioclavicular joint

- joint dislocations

- anatomy

- dissection

Objective To study the anatomy of the medial coracoclavicular ligament and assess the contribution of the acromioclavicular, coracoclavicular and medial coracoclavicular ligaments to the stability of the acromioclavicular joint.

Methods Twenty-six shoulders from 16 fresh cadavers were dissected after placement in dorsal recumbency with a 15-cm cushion between the shoulder blades. An extended deltopectoral approach was performed proximally and medially, followed by plane dissection and ligament identification. The acromioclavicular and coracoclavicular distances were measured using points previously marked with a millimeter caliper. Six of these specimens were submitted to a biomechanical study. The acromioclavicular ligament, the coracoclavicular ligament and the medial coracoclavicular ligament were sectioned sequentially, and a cephalic force of $20 \mathrm{~N}$ was applied to the lateral clavicle. The acromioclavicular and coracoclavicular distances were measured in each of the ligament section stages.

Results The right medial coracoclavicular ligament presented, on average, $48.9 \mathrm{~mm}$ in length and $18.3 \mathrm{~mm}$ in width. On the left side, its mean length was $48.65 \mathrm{~mm}$, with a mean width of $17.3 \mathrm{~mm}$. Acromioclavicular, coracoclavicular and medial coracoclavicular ligament section resulted in a statistically significant increase in the coracoclavicular distance and posterior scapular displacement.

Conclusion The medial coracoclavicular ligament is a true ligamentous structure found in all dissected shoulders. Our results showed that the scapular protraction relaxed the medial coracoclavicular ligament, while scapular retraction tensioned it; in addition, our findings demonstrate that this ligament contributes to the vertical and horizontal stability of the acromioclavicular joint.
\end{abstract}

coracoclavicular medial houve aumento estatisticamente significativo da distância córaco-clavicular e um deslocamento posterior da escápula.

Conclusão O ligamento coracoclavicular medial é uma estrutura ligamentar verdadeira, presente em todos os ombros dissecados. Nossos resultados demonstraram que o ligamento coracoclavicular medial encontra-se relaxado com a escápula em protração e tenso com a escápula em retração e segundo nossos resultados participa tanto da estabilidade vertical quanto da estabilidade horizontal da articulação acromioclavicular.

\section{Introdução}

A literatura sobre a anatomia e a biomecânica ligamentar da articulação acromioclavicular (AC) é muito rica e há inúmeros trabalhos que discutem os diversos tipos de tratamento das lesões traumáticas desta articulação, suas abordagens cirúrgicas abertas e artroscópicas, considerando-se os ligamentos acromioclaviculares e coracoclaviculares laterais (conoide e trapezoide). No entanto, raros são os artigos que discutem a anatomia ligamentar medial ao processo coracoide, notadamente do ligamento coracoclavicular medial (LCCM) e a importância desse ligamento na luxação acromioclavicular, como neste estudo anatômico.

Descrito como "Ligamento Bicorne" por apresentar dois fascículos principais em 1802 por Leopoldo Caldani, ${ }^{1}$ o LCCM também já foi considerado um espessamento da fáscia clavipeitoral, ${ }^{2}$ um ligamento coracocostal ${ }^{3}$ e até mesmo já teve a sua existência negada. ${ }^{4-8}$

O LCCM forma uma faixa de fibras de cor amarelo perolada que se origina da borda medial do processo coracoide por meio de dois fascículos (anterior e posterior), os quais se fundem para formar o corpo ligamentar e insere-se na porção medial da clavícula. ${ }^{2,9,10}$ Existem algumas divergências em relação à sua inserção. Alguns autores ${ }^{11-13}$ dividem-na em dois fascículos (superior, na borda anterior da clavícula e inferior, na borda esternal da primeira costela). ${ }^{10}$ Para outros autores, a sua inserção ocorre na borda anterior da clavícula, dividida em três expansões (superior, inferior e medial).

Recentemente foram descritas as características do LCCM em imagens de Ressonância Nuclear Magnética. ${ }^{14}$

Estudamos a anatomia do LCCM com o objetivo de avaliar a sua função e verificar a sua contribuição na estabilidade da articulação acromioclavicular nos casos de lesão da mesma.

\section{Material e métodos}

Esse estudo foi aprovado pelo Comitê de Ética em Pesquisa da nossa instituição (número CAAE: 12358919.0.0000.5463, e realizado no Serviço de Verificação de Óbitos da Capital (SVOC) da Universidade de São Paulo (USP). 


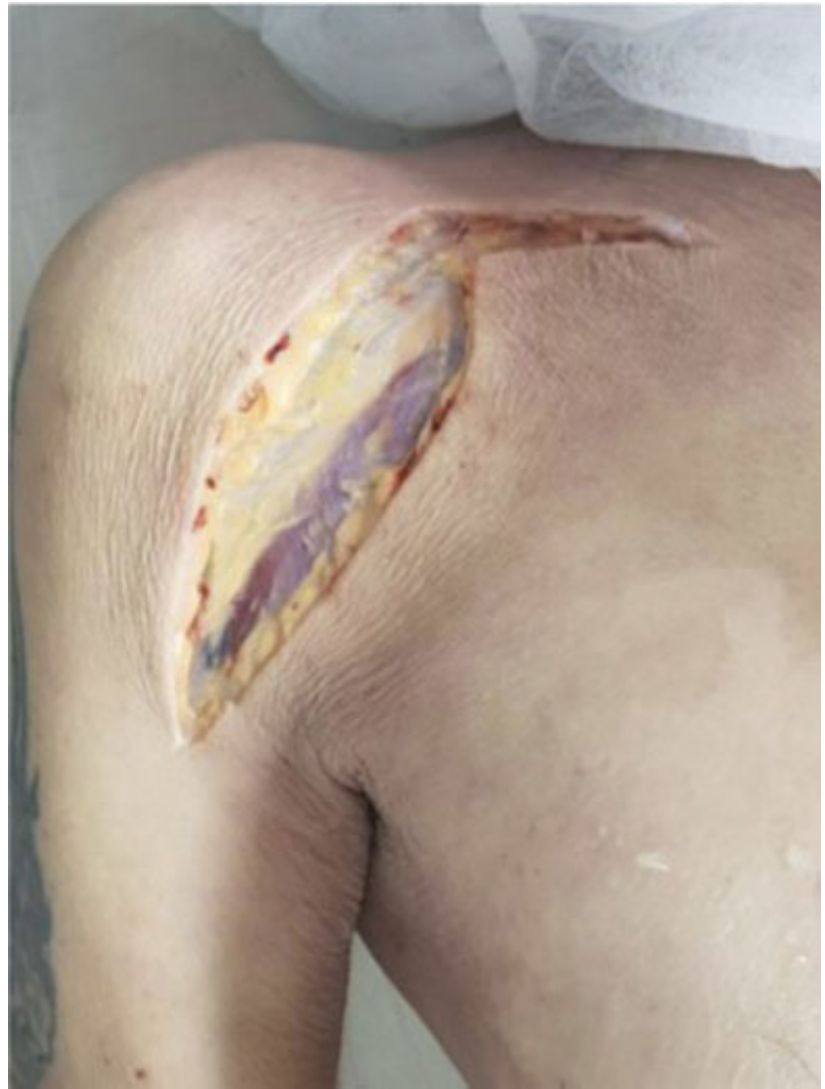

Fig. 1 Via deltopeitoral estendida.

Dissecaram-se 26 ombros de 16 cadáveres frescos adultos sem cicatrizes ou deformidades prévias. Todas as dissecções foram feitas pelo mesmo grupo de pesquisadores, ortopedistas especialistas em cirurgia do ombro e de forma padronizada, com o cadáver na posição de decúbito dorsal horizontal com um coxim de $15 \mathrm{~cm}$ de altura na região interescapular. Iniciou-se a dissecção com uma via deltopeitoral estendida proximal e em "L", seguindo horizontalmente na direção da articulação esterno clavicular ( - Figura 1 - Via).

Realizou-se dissecção por planos, desinseriram-se os músculos deltoide e peitoral maior da face anterior da clavícula e identificou-se o ligamento coracoclavicular medial desde a sua inserção no processo coracoide até sua inserção na extremidade medial da clavícula; e mediram-se seu comprimento e sua largura com paquímetro milimetrado (-Figuras 2 e 3). Realizaram-se os movimentos de retração e de protração da escápula observando- se o efeito na tensão do ligamento. Seccionaram-se, nesta ordem, os ligamentos acromioclaviculares, trapezóide, conóide e o ligamento coracoclavicular medial e observando-se o deslocamento da articulação acromioclavicular tanto no sentido cefálico quanto no anteroposterior (-Figura 4).

Na primeira parte do trabalho foi realizado um estudo anatômico em 20 ombros de 10 cadáveres para avaliação da morfologia ligamentar do LCCM. Já na segunda parte foi realizado um estudo biomecânico com seis ombros de seis cadáveres, onde foi realizado o seccionamento sequencial dos ligamentos em seis ombros, através da tração cefálica da clavícula, com força de tração constante de $20 \mathrm{~N}$, aplicada a

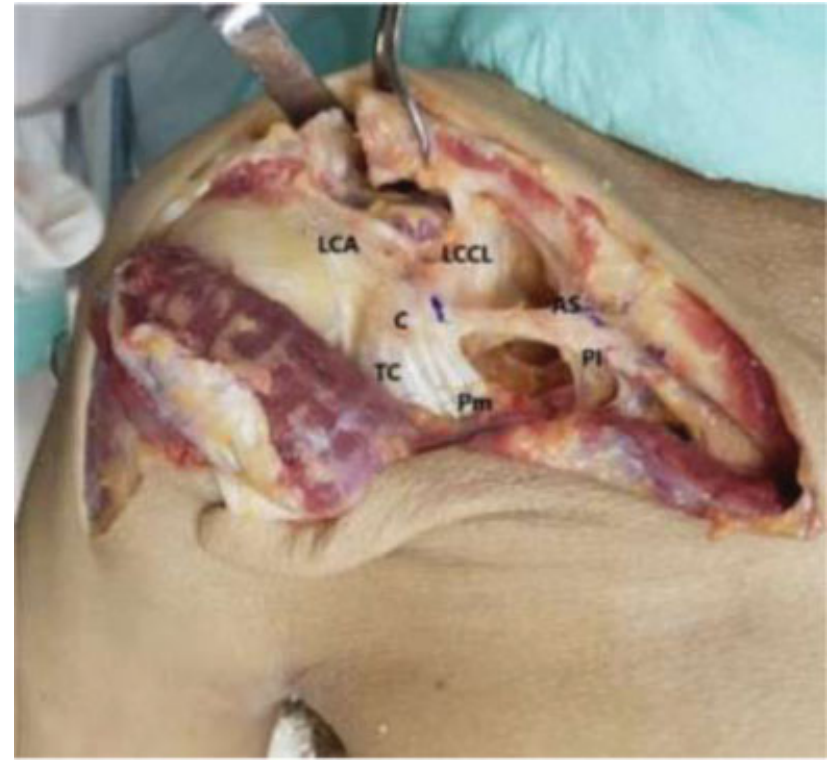

Fig. 2 Dissecção, identificação das estruturas e medição do LCCM (Ligamento coracoclavicular medial); LCA: ligamento coracoacromial; C: coracóide; TC: tendão conjunto; Pm: peitoral menor; LCCL: ligamentos coracoclaviculares laterais; AS: banda anterossuperior; PI: banda posteroinferior.

$1 \mathrm{~cm}$ da borda distal e medida por dinamômetro. A unidade $\mathrm{kgF}$ é considerada equivalente à unidade Newton. Foram marcados 3 pontos anatómicos $(A, B, C)$ sendo o ponto $A$ localizado a $1 \mathrm{~cm}$ do ponto mais medial do acrômio; o ponto $B$ sendo $1 \mathrm{~cm}$ do ponto mais lateral da clavícula e o ponto $C$ localizado na borda lateral do processo coracoide. A dissecção da articulação AC e a transecção de seus ligamentos intrínsecos foram realizadas pela primeira vez; depois, uma secção sequencial dos ligamentos trapezoide e conoide seguidos pelo LCCM. A cada passo, foi aplicada uma força

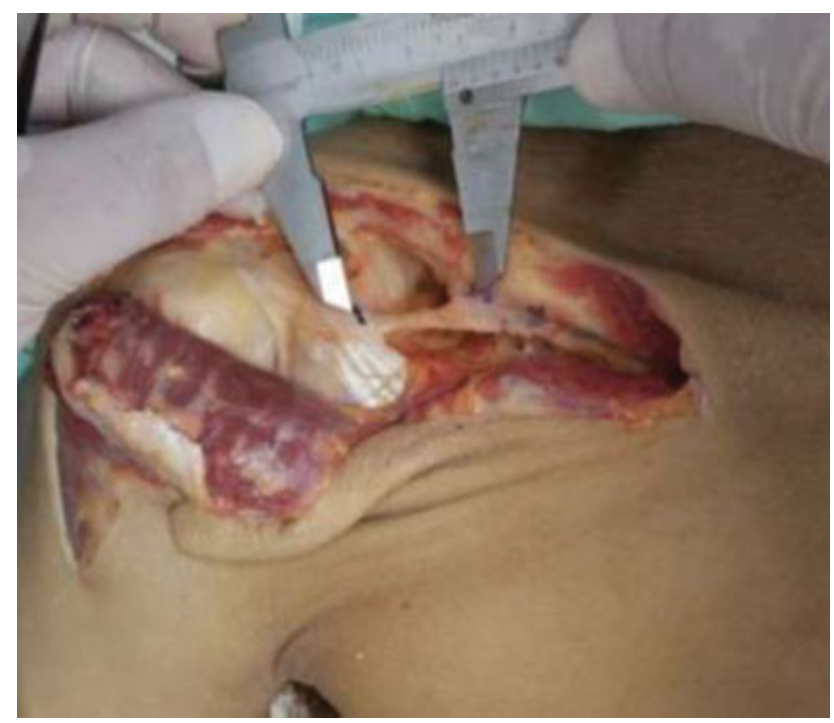

Fig. 3 Dissecção, identificação das estruturas e medição do LCCM (Ligamento coracoclavicular medial); LCA: ligamento coracoacromial; C: coracóide; TC: tendão conjunto; Pm: peitoral menor; LCCL: ligamentos coracoclaviculares laterais; AS: banda anterossuperior; PI: banda posteroinferior. 


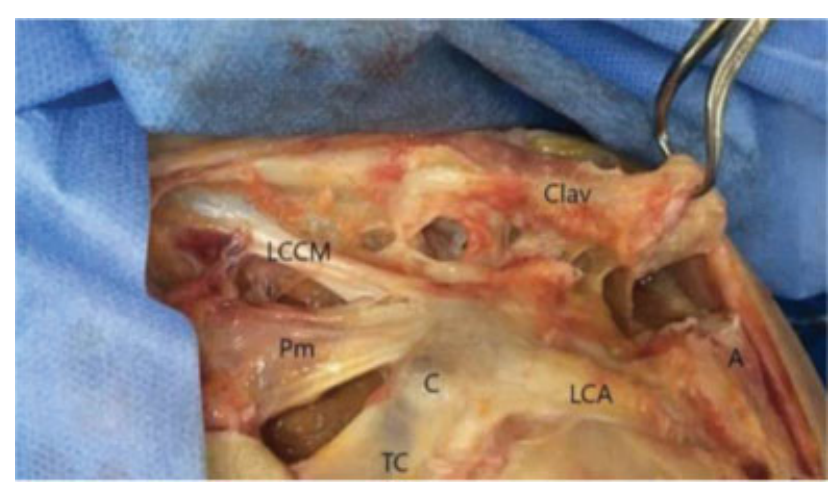

Fig. 4 Dissecção e identificação das estruturas. Clav: clavícula; A: acrômio; LCCM: Ligamento coracoclavicular medial; LCA: ligamento coracoacromial; C: coracóide; TC: tendão conjunto; Pm: peitoral menor.

de tração uniforme $20 \mathrm{~N}$ na extremidade lateral da clavícula, a distância vertical e horizontal entre o acrômio e a clavícula (da borda superior do acrômio até a borda superior da clavícula distal) e a distância vertical entre o coracoide e a clavícula (espaço coracoclavicular) e entre o acrômio e a clavícula foi medida após cada corte, conforme descrito por Moya et al. ${ }^{15}$

Foi realizada análise descritiva dos dados, apresentadas através de média e desvio padrão. As diferenças das variâncias foram verificadas por meio da Análise da Variância (ANOVA) com medidas repetidas. Os resultados foram considerados estatisticamente significativos aqueles cujo valor de $\mathrm{p}$ foi inferior a 0,05 , sempre considerando hipóteses alternativas bicaudais. As informações coletadas formaram um banco de dados desenvolvido no programa Excel ${ }^{\circledR}$ for Windows e a análise estatística foi realizada através do software SPSS ${ }^{2} 16.0$.

\section{Resultados}

O ligamento coracoclavicular medial foi identificado e mensurado em todos os ombros dissecados. No ombro direito, encontramos uma média de comprimento de 48,9mm (variando de 46 a $53 \mathrm{~mm}$ ) e uma média de largura de $18,3 \mathrm{~mm}$ (variando de 17 a $20 \mathrm{~mm}$ ). No ombro esquerdo encontramos uma média de comprimento de $48,65 \mathrm{~mm}$ (variando de 45,5 a $52,5 \mathrm{~mm}$ ) e uma média de largura de $17,3 \mathrm{~mm}$ (variando de 16
Tabela 1 Resultado das medidas do LCCM

\begin{tabular}{|l|l|l|l|}
\hline & Média & $\begin{array}{l}\text { Desvio } \\
\text { Padrão }\end{array}$ & Variância \\
\hline $\begin{array}{l}\text { Comprimento } \\
\text { LCCMedial D }\end{array}$ & $48,90 \mathrm{~mm}$ & 2,438 & 5,933 \\
\hline $\begin{array}{l}\text { Comprimento } \\
\text { LCCMedial E }\end{array}$ & $48,65 \mathrm{~mm}$ & 2,7262 & 7,432 \\
\hline Largura LCCMedial D & $18,30 \mathrm{~mm}$ & 0,949 & 0,900 \\
\hline Largura LCCMedial E & $17,30 \mathrm{~mm}$ & 0,949 & 0,900 \\
\hline
\end{tabular}

a $18 \mathrm{~mm})$. O resultado da análise estatística está descrito na - Tabela 1.

Dividiu-se o LCCM em três segmentos: origem, corpo e inserção. A origem deste ligamento situa-se na borda medial do processo coracoide, medial e posterior à inserção do músculo peitoral menor e distal à origem dos ligamentos conoide e trapezoide. O LCCM é bifurcado em sua origem e possui dois fascículos, um anterior e outro posterior, os quais se fundem para formar o corpo ligamentar, de cor amarelo perolada e consistência fibro-elástica, que corre anteriormente ao músculo subclávio. A sua inserção situa-se na borda ântero-inferior da clavícula medial, em uma topografia que, devido à grande quantidade de aponeurose, dificulta o seu isolamento.

Durante o movimento de protração da escápula houve o relaxamento do LCCM e durante o movimento de retração das escápulas ocorreu o tensionamento deste ligamento.

Em seis dessas amostras, seccionaram-se, nesta ordem, os ligamentos acromioclaviculares, trapezoide, conoide e o LCCM, observando-se o deslocamento superior da articulação acromioclavicular e posteriorização da escápula. Uma força cefálica de $20 \mathrm{~N}$ foi aplicada na clavícula lateral com o uso dinamômetro analógico portátil, registrando a distância da AC e o espaço coracoclavicular e sua variação. Avaliamos também o movimento anteroposterior da clavícula seguindo a seção MCCL. Todos do sexo masculino (100\%). O lado direito foi observado em $66,7 \%$ dos casos. O IMC médio foi de 23,8 , com desvio padrão de 5,6, variando entre 12,5 e 32,0. A média de idade encontrada foi de 67 anos, com desvio padrão de 9,5, variando entre 58 e 84 anos (-Tabela 2).

Tabela 2 Lista das medidas encontradas nos cadáveres avaliados

\begin{tabular}{|c|c|c|c|c|c|c|c|c|c|c|c|c|c|c|c|}
\hline \multirow[t]{2}{*}{ Cad } & \multirow[t]{2}{*}{ Altura } & \multirow[t]{2}{*}{ Idade } & \multirow[t]{2}{*}{ Lado } & \multicolumn{4}{|c|}{$\begin{array}{l}\text { Distancia Acromio Clavi- } \\
\text { cular }\end{array}$} & \multicolumn{4}{|c|}{$\begin{array}{l}\text { Distancia Acromio Clavi- } \\
\text { cula Posterior }\end{array}$} & \multicolumn{4}{|c|}{$\begin{array}{l}\text { Distancia Coracoide Cla- } \\
\text { vicula }\end{array}$} \\
\hline & & & & 1 & 2 & 3 & 4 & 1 & 2 & 3 & 4 & 1 & 2 & 3 & 4 \\
\hline 1 & 1,85 & 68. & $\mathrm{D}$ & 0,8 & 1,6 & 2,5 & 3,0 & 2,0 & 2,9 & 3,1 & 3,2 & 3,4 & 4,0 & 5,2 & 5,7 \\
\hline 2 & 1,75 & 70. & $\mathrm{D}$ & 0,6 & 1,4 & 3,0 & 3,4 & 2,2 & 2,3 & 3,8 & 4,5 & 3,0 & 3,5 & 4,8 & 5,5 \\
\hline 3 & 1,65 & 64. & $\mathrm{D}$ & 0,9 & 1,4 & 2,2 & 2,7 & 2,2 & 2,5 & 2,7 & 3,5 & 3,3 & 3,8 & 4,5 & 5,0 \\
\hline 4 & 1,87 & 58. & $\mathrm{D}$ & 0,6 & 1,7 & 2,6 & 2,9 & 2,8 & 4,0 & 4,5 & 4,6 & 3,3 & 4,3 & 5,3 & 5,5 \\
\hline 5 & 1,53 & 59. & $\mathrm{E}$ & 1,0 & 2,1 & 3,2 & 3,9 & 1,8 & 2,5 & 3,9 & 4,3 & 3,5 & 4,0 & 5,0 & 5,8 \\
\hline 6 & 1,70 & 84. & $E$ & 0,2 & 1,2 & 2,1 & 2,2 & 3,6 & 5,1 & 5,1 & 5,2 & 2,8 & 4,0 & 5,0 & 5,4 \\
\hline
\end{tabular}

Legenda: Cad- cadaver; 1- sem lesao; 2- Lesao AC; 3- Lesao CC; 4-Lesao CCM. 


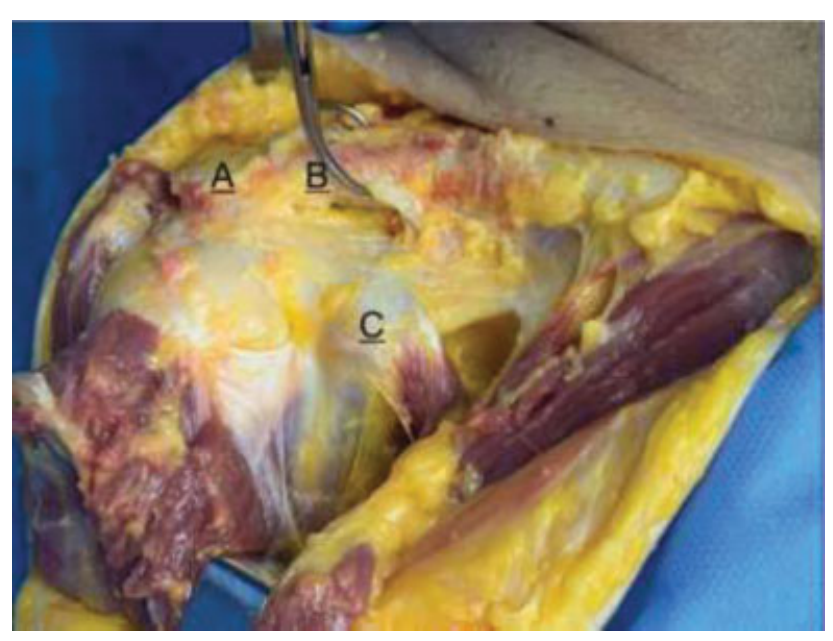

Fig. 5 Pontos A,B e C demarcados.

Avaliamos o deslocamento superior através das distancias AB (acrômio-clavicula) e BC (coracoide-clavícula); deslocamento posterior através da distancia $A B$ (acrômio-clavicula) posterior. Ao medir o deslocamento da clavícula no ponto $A B$ superior, no ponto $A B$ posterior e no ponto $B C$ em quatro momentos distintos (sem lesão dos ligamentos acromioclaviculares $[A C]$ e coracoclaviculares $[C C]$ ), lesão $A C$, lesão $\mathrm{AC}+\mathrm{CC}$ e lesão AC + CC + LCCM (-Figuras 5 e $\mathbf{6}$ ), observouse, em todos os pontos de medicação, um aumento significativo do deslocamento, com significância estatística, conforme - Tabela 3 abaixo:

A distância média acrômio-clavícula $(A B)$ passou de $0,7 \mathrm{~cm}$ sem lesão a 3,0cm após secção de todos os ligamentos inclusive LCCM. Já a distância coracoide-clavícula (BC) média passou de $3,2 \mathrm{~cm}$ sem lesão para $5,5 \mathrm{~cm}$ com toda $\mathrm{sec} c ̧ a ̃ o$ ligamentar, com $\mathrm{p}<0,001$.

Com a secção ligamentar observamos um desvio posterior da escápula que se exacerbou após secção do LCCM.

\section{Discussão}

O ligamento coracoclavicular medial, também denominado ligamento coracoclavicular anterior de Henle e ligamento coracoclavicular horizontal de Soulié ${ }^{1,10,12-14}$ é motivo de controvérsias e há até autores que não o citam na anatomia ligamentar do coracoide. ${ }^{16}$ Klassen et al. ${ }^{8}$ não o encontraram nas suas dissecções; Rouvière ${ }^{2}$; Vallois e Thomas $^{13}$ não o consideraram uma estrutura própria, mas um espessamento de estruturas vizinhas, isto é, um cordão da fáscia clavipei-

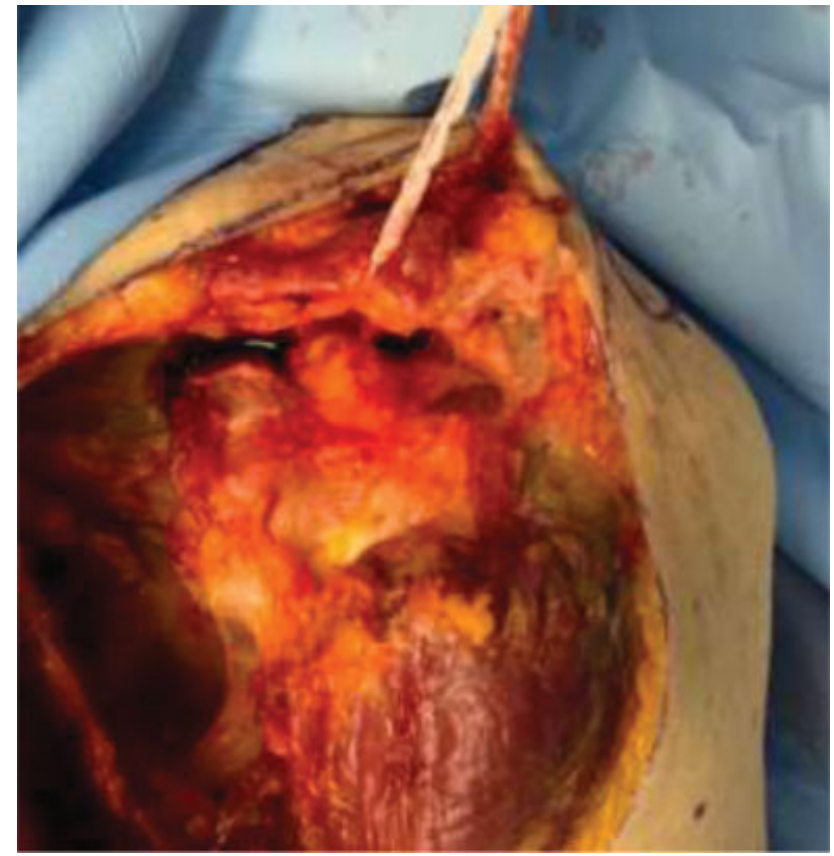

Fig. 6 Realização de tração de $20 \mathrm{~N}$ após secção dos ligamentos.

toral. ${ }^{17}$ Terra et al. ${ }^{18}$ descrevem o ligamento coracoclavicular medial como uma variação anatômica do ligamento conoide.

Poitevin et al., ${ }^{10}$ descreveram a presença do LCCM em cem por cento dos ombros estudados (15 adultos e 8 fetos). Para estes autores o LCCM tem sua origem no processo coracoide, dividido em dois fascículos e sua inserção na clavícula medial, dividida em três expansões (superior, inferior e medial).

O LCCM também já foi objeto de estudo por imagem de ressonância magnética e visibilizado em toda sua extensão, desde a sua origem no processo coracoide até a sua inserção na clavícula medial, com a descrição imaginológica de suas características anatômicas. ${ }^{14}$

Stimec et al. ${ }^{12}$ descreveram o ligamento a partir da dissecção de um espécime de cadáver do sexo feminino de 92 anos de idade. Os autores ressecaram o ligamento para sua mensuração e análise histológica e confirmaram a sua natureza ligamentar, a sua estrutura e a disposição das fibras colágenas, pareadas e rodeadas por uma fina camada de tecido conjuntivo rico em vascularização, à semelhança do ligamento conoide.

Ao iniciarmos os nossos estudos do LCCM verificamos existir pouquíssima literatura atual e que Poitevin et al., ${ }^{10}$ Moyá et al. ${ }^{15}$ e Azulay et al. ${ }^{14}$ apresentavam estudos recentes.

Tabela 3 Média das distâncias dos pontos e desvio padrão

\begin{tabular}{|l|l|l|l|l|l|}
\hline & Sem Lesao & Lesao AC & Lesao CC & Lesao CCM & P \\
\hline & Media \pm DP & Media \pm DP & Media \pm DP & Media \pm DP & \\
\hline Distancia AB & $0,7 \pm 0,3$ & $1,6 \pm 0,3$ & $2,6 \pm 0,4$ & $3,0 \pm 0,6$ & $<0,001$ \\
\hline Distancia AB Post & $2,4 \pm 0,7$ & $3,2 \pm 1,1$ & $3,9 \pm 0,9$ & $4,2 \pm 0,7$ & 0,009 \\
\hline Distancia BC & $3,2 \pm 0,3$ & $3,9 \pm 0,3$ & $5,0 \pm 0,3$ & $5,5 \pm 0,3$ & $<0,001$ \\
\hline
\end{tabular}


Procuramos utilizar metodologia semelhante pois assim poderíamos confrontar nossos resultados, discutir, concordar e ou discordar de suas conclusões. Em nossas descrições os valores absolutos encontrados para aferição do comprimento ligamentar foi semelhante ao encontrado por outros autores em suas dissecções.

Em nosso estudo, o LCCM foi encontrado em todas as dissecções, de forma bilateralmente, assim como descrito por outros autores. Observou- se que a sua inserção na clavícula medial se situa na porção ântero-inferior deste osso, porém é cercada de aponeuroses que podem ser confundidas com ramificações ou expansões deste ligamento. Observou-se ainda que este apresenta íntimo contato com o músculo subclávio, achado que poderia levar à conclusão de que seria resultado de uma metaplasia muscular escápulo-torácica, tal como descrito por Luk et al. ${ }^{19}$ No entanto, descartamos esta hipótese posto que este ligamento foi descrito em dissecções de fetos humanos. ${ }^{10}$ Moya et al. ${ }^{15}$ sugerem que o LCCM é uma estrutura constante com características mecânicas de um ligamento que pode atuar como a última restrição do espaço coracoclavicular tanto em direção cefálica quanto posterior, impedindo um deslocamento adicional na ausência dos ligamentos coracoclaviculares laterais.

Como o estudo é realizado no Serviço de Verificação de Óbitos da Capital em cadáveres frescos e não em peças anatômicas como Moya et al. ${ }^{15}$ optamos por posicionar o cadáver na posição de decúbito dorsal horizontal com um coxim de $15 \mathrm{~cm}$ de altura na região interescapular pois nesta posição o ombro esta livre e não mantem contato com a mesa de dissecção com isso tem-se uma melhor avaliação das articulações envolvidas após secção ligamentar progressiva.

Observou-se neste estudo o relaxamento do LCCM durante a protração escapular ipsilateral e o tensionamento deste ligamento durante a retração escapular, levando-nos a levantar a hipótese de que o LCCM apresenta uma função de um estabilizador horizontal secundário da articulação acromioclavicular. Reforça esta hipótese a observação de que, após a secção, nesta ordem, dos ligamentos acromioclaviculares, trapezoide e conoide, e mantendo o cadáver com um coxim interescapular e os ombros suspensos, a excisão do LCCM na sua origem no processo coracoide promove um aumento significativo da distância acromioclavicular, com significância estatística.

Constatamos também que ao seccionar todos os ligamentos que unem a escápula a clavícula o membro superior fica totalmente sujeito as forças gravitacionais, isto é, a escápula inferioriza em relação a clavícula, criando uma imagem semelhante de um paciente em ortostase com luxação acromioclavicular estágio 5 de Rockwood. ${ }^{16}$ Estas observações permitem-nos supor que a lesão do LCCM ocorra nos estágios 4 e 5 da classificação de Rockwood para as luxações acromioclaviculares.

Entendemos que o conhecimento das estruturas ligamentares mediais ao processo coracoide podem contribuir para o entendimento das lesões traumáticas acromioclaviculares, assim como pode ser de grande valia nas abordagens cirúrgicas que necessitem de dissecção das partes moles inseridas no processo coracoide, como as transferências musculares, as cirurgias de Bristow e Latarjet e as descompressões na síndrome do desfiladeiro torácico.

A existência do LCCM como estrutura anatômica está bem estabelecida. No entanto acreditamos que, novos estudos biomecânicos e de imagem podem esclarecer ainda mais sua função estabilizadora da articulação acromioclavicular.

Uma das deficiências ou limitação do nosso estudo é que este foi realizado em cadáveres com o dorso elevado em somente 15 graus. O ideal seria com maior flexão do corpo para melhor avaliação da articulação acromioclavicular com auxílio da força gravitacional e ortostatismo. Outra limitação é o pequeno número de espécimes. No entanto, nossos achados são importantes para o conhecimento das estruturas envolvidas na luxação acromioclavicular e a função do LCCM na estabilidade dessa articulação. São necessários estudos que avaliem clinicamente a importância desse ligamento, correlacionando o exame clinico, radiográfico e com a ressonância nuclear magnética.

\section{Conclusão}

O ligamento coracoclavicular medial é uma estrutura ligamentar verdadeira, presente em todos os ombros dissecados. Encontra-se relaxado com a escápula em protração e tenso com a escápula em retração e participa tanto da estabilidade vertical e quanto da estabilidade horizontal da articulação acromioclavicular.

\section{Suporte Financeiro}

Não houve suporte financeiro de fontes públicas, comerciais, ou sem fins lucrativos.

Conflito de Interesses

Os autores declaram não haver conflitos de interesse.

\section{Referências}

1 Caldani LMA. Iconum anatomicarum explicatio. Ossa 1802;1:244

2 Rouvière H. Anatomie Humaine. 2nd ed. Paris: Masson; 1927

3 Williams PL, Bannister LH, Berry MM, , et al. Gray's Anatomy. 38th ed. Churchill Edinburgh: Livingstone; 1995

4 Ockert B, Braunstein V, Sprecher C, Shinohara Y, Kirchhoff C, Milz $\mathrm{S}$. Attachment sites of the coracoclavicular ligaments are characterized by fibrocartilage differentiation: a study on human cadaveric tissue. Scand J Med Sci Sports 2012;22(01):12-17

5 Salzmann GM, Paul J, Sandmann GH, Imhoff AB, Schöttle PB. The coracoidal insertion of the coracoclavicular ligaments: an anatomic study. Am J Sports Med 2008;36(12):2392-2397

6 Stine IA, Vangsness CT Jr. Analysis of the capsule and ligament insertions about the acromioclavicular joint: a cadaveric study. Arthroscopy 2009;25(09):968-974

7 Dal Molin DC, Ribeiro FR, Brasil Filho R, , et al. Via de acesso cirúrgico posterossuperior para o tratamento das luxações acromioclaviculares: resultados de 84 casos operados. Rev Bras Ortop 2012;47(05):563-567

8 Klassen JF, Morrey BF, Ann KN. Surgical anatomy and function of the acromioclavicular and coracoclavicular ligaments. Oper Tech Sports Med 1997;5(02):60-64

9 Stedman TL. A Practical Medical Dictionary. New York: W. Wood and Company; 1920 
10 Poitevin LA, Postan D, Moya D, , et al. Anatomía del ligamento córaco-clavicular medial. Primera parte: investigación anatómica. Rev Arg Anat 2014;5(04):119-126

11 Souteyrand-Boulanger J. Les formations fibreuses et les ligaments du triangle clavi- coraco- pectoral chez les primates. Mammalia 1966;30:645-666

12 Stimec BV, Lädermann A, Wohlwend A, Fasel JH. Medial coracoclavicular ligament revisited: an anatomic study and review of the literature. Arch Orthop Trauma Surg 2012;132(08):1071-1075

13 Vallois HV, Thomas L. Les formations fibreuses du triangle clavipectoral. Arch Anat Histol Embryol 1942;3:363-396

14 Azulay G, Mamone A, Moya D, Poitevin L, Postan D. Identificación del ligamento coracoclavicular medial o de Caldani en imágenes de resonancia magnética. Protocolo original y aplicaciones clínicas. Rev Asoc Argent Ortop Traumatol 2017;82(01):71-77
15 Moya D, Poitevin LA, Postan D, , et al. The medial coracoclavicular ligament: anatomy, biomechanics,and clinical relevance-a research study. JSES Open Access 2018;2(04):183-189

16 Rockwood CA Jr, Matsen FA, Wirth MA, Lippitt SB. The Shoulder. 4th ed. Philadelphia: W.B. Saunders; 2009

17 Martin RM, Vyas NM, Sedlmayr JC, Wisco JJ. Bilateral variation of subclavius muscle resembling subclavius posticus. Surg Radiol Anat 2008;30(02):171-174

18 Terra BB, Figueiredo EA, Marczk CS, , et al. Variação anatômica do ligamento coracoclavicular. RBM Rev Bras Med 2010;67(Suppl 6):17-20

19 Luk KD, Ho HC, Leong JC. The iliolumbar ligament. A study of its anatomy, development and clinical significance. J Bone Joint Surg Br 1986;68(02):197-200 